\title{
Future Missions to the Giant Planets that can Advance Atmospheric Science Objectives
}

Mark D. Hofstadter ${ }^{1 *}$, Leigh N. Fletcher ${ }^{2}$, Amy A. Simon ${ }^{3}$, Adam Masters ${ }^{4}$, Diego Turrini ${ }^{5}$, and Christopher S. Arridge ${ }^{6}$

${ }^{1}$ Jet Propulsion Laboratory, California Institute of Technology

Mail Stop 183-301

4800 Oak Grove Drive

Pasadena, CA 91109

USA

${ }^{2}$ University of Leicester

Department of Physics and Astronomy

University Road

Leicester

LE1 7RH

United Kingdom

${ }^{3}$ Goddard Space Flight Center

Greenbelt, MD 20771

USA

${ }^{4}$ The Blackett Laboratory, Imperial College London

Prince Consort Road

London

SW7 2AZ

United Kingdom

${ }^{5}$ Institute for Space Astrophysics and Planetology INAF-IAPS

Via Fosso del Cavaliere 100

00133 Rome

Italy

${ }^{6}$ Lancaster University

Lancaster

LA1 4YW

United Kingdom

*Corresponding author

E-mail: Mark.Hofstadter@jpl.nasa.gov

Phone: +1 818-354-6160 


\begin{abstract}
Other papers in this special issue have discussed the diversity of planetary atmospheres and some of the key science questions for giant planet atmospheres to be addressed in the future. There are crucial measurements that can only be made by orbiters of giant planets and probes dropped into their atmospheres. To help the community be more effective developers of missions and users of data products, we summarize how NASA and ESA categorize their planetary space missions, and the restrictions and requirements placed on each category. We then discuss the atmospheric goals to be addressed by currently approved giant-planet missions as well as missions likely to be considered in the next few years, such as a joint NASA/ESA Ice Giant orbiter with atmospheric probe. Our focus is on interplanetary spacecraft, but we acknowledge the crucial role to be played by ground-based and near-Earth telescopes, as well as theoretical and laboratory work.
\end{abstract}

Keywords: Spacecraft, missions, giant planets, Ice Giants, Gas Giants, Atmospheres

\title{
Acknowledgements:
}

Fletcher was supported by a Royal Society Research Fellowship and European Research Council Consolidator Grant (under the European Union's Horizon 2020 research and innovation program, grant agreement No 723890) at the University of Leicester. Masters was supported by a Royal Society University Research Fellowship. Hofstadter's work was carried out at the Jet Propulsion Laboratory, California Institute of Technology, under a contract with the National Aeronautics and Space Administration (NASA). 


\section{Introduction}

Earlier papers in this special issue (the result of a workshop titled "The Diversity of Planetary Atmospheres" hosted by the International Space Science Institute in Bern, Switzerland during November of 2018) have laid out the nature of the planetary atmospheres we know of andto the limits of our understanding - their histories and the important physical processes at work (e.g. Gaillard et al. 2019; Mills et al. 2019; Fletcher et al. 2019; Showman 2019; Leconte et al. 2019). They have also speculated on atmospheres yet-to-be discovered. In each case, key science questions were identified. This paper does not review those science discussions, but instead discusses the space missions to the Giant Planets that are likely to dramatically advance our understanding of the diversity of giant planet atmospheres. Our goal is to review what the currently planned giant planet missions can achieve, and outline the opportunities for future missions and some of the constraints upon them. In this way we hope to help the scientific community anticipate what atmospheric data sets will be available, help them provide useful inputs to the planning process of space agencies, and also help the community identify measurement gaps that could trigger ideas for future mission proposals.

While our focus is exclusively on what can be achieved with interplanetary robotic spacecraft, this is not meant to deny the critical role ground- and near Earth-based observations, laboratory work, and theory will play in improving our understanding of giant planets and their atmospheres.

\section{Why Spacecraft are Necessary}

There are restrictions on measurements made from the Earth and even from space-based telescopes near the Earth, which limit their ability to probe giant planet atmospheres. These limitations include viewing geometry, spatial resolution, and (for ground-based observations) wavelength coverage. Furthermore, there are several crucial measurements that can only be made in situ from a spacecraft sent to visit these worlds. For these reasons, spacecraft will play a central role in advancing our understanding of planetary atmospheres.

Observations of the giant planets from an Earth- or near-Earth based vantage point are fundamentally limited to remote sensing, exploiting physical and chemical phenomena that alter the emergent spectrum of light across a vast swathe of the electromagnetic spectrum, from the radio through the IR, visible, and UV out to X-rays. Ground-based facilities, ranging from the 3-to-10-m diameter visible/near-IR observatories to radio antenna arrays distributed over tens of kilometers, are currently capable of monitoring atmospheric, ionospheric, and auroral processes on each of the four giants. These observations are mostly limited to the sunlit summer hemispheres, however, as the winter hemispheres are by definition tilted away from the Sun and the inner solar system. For example, Neptune's northern winter high latitudes have never been observed, either from the Voyager-2 flyby in 1989, or from Hubble and ground-based observations in the ensuing decades. Furthermore, all of these Earth-based observations are at low phase angles, such that there is no possibility of observing the terminators or nightside of these worlds (we use the term Earthbased to include facilities on the ground and operating in near-Earth space). Thus, Earthbased observations cannot fully explore diurnal and seasonal variations in giant planet 
atmospheres, probe the global energy balance of the planets, or constrain cloud properties by observing them over a wide range of solar phase angles.

And while the size of Earth-based telescopes is growing ever larger, improving their spatial resolution, they cannot compete in spatial resolution, time resolution, or sensitivity with the close-in views afforded by a visiting spacecraft. This makes a nearby spacecraft the best platform when trying to determine the abundance of trace species in the atmosphere (Mills et al. 2019), or to observe atmospheric dynamics (see papers by Fletcher et al., Kaspi et al., and by Showman in this issue). Finally, ground-based remote sensing observations are limited by absorption in Earth's atmosphere which prevents measurements at X-ray and most UV wavelengths, and severely limits coverage in the infrared and near-IR. Even where the atmosphere is relatively transparent measurements are subject to variability caused by the changing column of terrestrial air between the observer and the giant planet target.

The most compelling argument for sending spacecraft to the giant planets, however, is that there are some measurements which cannot be made at all from the inner solar system, and absolutely require an in-situ spacecraft. The best example of this is measuring the abundances of noble gases and their isotopic ratios. These measurements are the most reliable indicators we have of the planetary formation process and subsequent evolution (see Venturini et al. 2019), but the non-reactive nature of these species requires them to be measured by an atmospheric entry probe. This is why such probes have always been a high priority at the giant planets. Entry probes are also needed to reliably measure the abundances and isotopic ratios of species that can condense in the atmosphere and form clouds, such as $\mathrm{NH}_{3}, \mathrm{CH}_{4}, \mathrm{H}_{2} \mathrm{O}$, and $\mathrm{H}_{2} \mathrm{~S}$, and these species also constrain planetary formation models (e.g. Atreya et al. 2019; Mousis et al. 2018). Entry probes also provide essential ground truth measurements of temperature, density, aerosols, and composition as functions of altitude that validate remote sensing studies.

An orbiting spacecraft also provides important in situ measurements of the environment surrounding each giant planet, relevant to understanding their atmospheres. In situ measurements of the gravity field constrain the depths of zonal winds and other meteorological features (Kaspi et al. 2019) while magnetic field and exospheric composition measurements help us understand the atmospheric loss processes at work (Ramstad and Barabash 2019). In addition to measurements targeting a giant planet atmosphere, an in situ spacecraft provides a wealth of information about the entire system which can also deepen our understanding of atmospheric diversity. Some giant planet satellites have their own unique atmospheres (e.g. Titan in the Saturn system). The composition, structure, and dynamics of satellites and rings can provide clues about the formation and evolution of the host giant planet as well. Finally, the structure and evolution of giant planet magnetospheres may help us better understand how star-planet interactions can influence planetary atmospheres.

For all these reasons, sending spacecraft to the giant planets in our solar system, dropping probes into their (and their satellite!) atmospheres, and studying the complex interplay of plasmas, satellites, and rings around these planets, will play a central role in understanding atmospheres in and beyond our solar system. 


\section{The Categories of Possible Missions}

This section describes the existing framework within NASA and ESA that defines missions to the giant planets. It is meant to familiarize scientists who might propose a mission or instrument with the type of programmatic constraints under which they would work and some of the terminology commonly used. The respective space agencies must be consulted for the latest information and for details on specific programs. While NASA and ESA are the two agencies most likely to lead efforts in the outer solar system, we note that other nations may make significant contributions. A summary of NASA and ESA mission types is provided in Table 1.

\subsection{NASA Mission Categories}

NASA categorizes missions in two ways, how they are managed and their cost. The management structure (by which we mean how the mission is chosen, developed, and operated) is referred to as either Strategic or PI-led. For Strategic missions (also called Assigned missions) NASA uses inputs from the community and formal advisory groups to identify a high-priority planetary mission and assigns the design, development, operations, and management of that mission to one of its centers. Strategic missions are typically large, complex, and expensive, such as the Cassini mission to Saturn and the James Web Space Telescope. PI-led missions, also referred to as Competed missions, reflect more of a "bottom-up" approach, in which NASA announces an opportunity for a space mission, and accepts proposals from any qualified team to execute the mission. The announcement can call for a mission to a specific or from a limited list of targets, or it can be open to all ideas. Peer and management panels review the proposals, and NASA selects one or more for eventual flight based both on the reviews and programmatic considerations such as cost and balance among scientific disciplines. PI-led missions tend to be smaller than Strategic ones, and more limited in their scientific focus. The Juno mission to Jupiter is an example of a PIled mission. Note that even Assigned missions can have competed elements: for example, instruments are typically competed even though the mission as a whole is not. Similarly, a Competed mission may be required to include a specific instrument or technology in its design.

In addition to categorizing missions by how they are managed, NASA also categorizes missions by cost (Fig. 1). The dividing lines between cost categories can in principle change year-to-year, so all specific dollar amounts presented here should be considered approximate. NASA can also change what components are factored into a mission's official cost. For example, the launch vehicle is typically not included in the cost used to categorize missions. Furthermore, NASA may choose to provide certain technologies, instruments, or personnel at reduced or no cost to a mission as a way to encourage their use or otherwise advance NASA's strategic goals. An example of this is that NASA has recently excluded many mission operations costs (referred to as Phase-E costs) from the "cost" of PI-led missions. This was done to allow long-duration outer solar system missions to compete against potentially shorter-duration inner solar system missions within the lower-cost mission categories. While discussing budgets, it is worth pointing out that NASA typically includes instruments and science teams within the cost of the mission, whereas ESA typically considers its cost to be only for design, building, and operation of the spacecraft itself, while individual ESA member states pay for the instruments and their associated science teams. 
(See the ESA Mission Categories section for additional details.) One must be careful to always check how cost is defined when trying to compare missions, and note that the cost typically discussed may not reflect the true total expense of a mission that is flown.

Setting aside these budget details for the moment, the largest NASA missions, called Flagships, are the most expensive. Historically, Flagship missions have always been Assigned (Strategic), and they cost more than US\$1000M. Smaller missions are typically competed. Missions costing between US\$500M and US\$1000M are referred to as New Frontiers missions, and those costing less than US\$500M are called Discovery missions. There have not been any Discovery missions flown to a giant planet (though several have been proposed). Various factors, such as distance from the Sun and the long flight times to the outer solar system, make it challenging to design a giant-planet mission that fits within the Discovery category. The Juno mission is an example of a successful New Frontiers proposal to a giant planet. For even less expensive flight opportunities, those costing under US\$55M, NASA utilizes its SIMPLEx (Small Innovative Missions for Planetary Exploration) or Stand ALone Missions of Opportunity Notice (SALMON) programs. At that cost cap, it is unlikely NASA could lead a giant planet mission, but it is feasible for NASA to provide an instrument or flight element (e.g. SmallSat) to be carried by a different mission or agency.

Under NASA's current system, Flagship and Discovery missions may be flown to any solar system object. Proposals for New Frontiers missions, however, must choose one of a small number of targets, based on a list set by the Planetary Science Decadal Survey (National Research Council 2011). That list can be altered at NASA's discretion. In the most recent New Frontiers call (NF-4), the allowed New Frontiers missions were:

- Comet sample return,

- Lunar South Pole-Aitken Basin sample return,

- Saturn atmospheric probe,

- Trojan tour and rendezvous,

- Venus in-situ explorer,

- Exploration of an Ocean World (either Titan or Enceladus).

The Decadal Survey calls for adding two additional mission targets to the fifth New Frontiers call: an lo Observer, and a Lunar Geophysical Network. At the time of this writing NASA's specific plans for NF-5 are not known.

On average, NASA begins one or two Flagships, two New Frontier, and 5 Discovery missions per decade. 


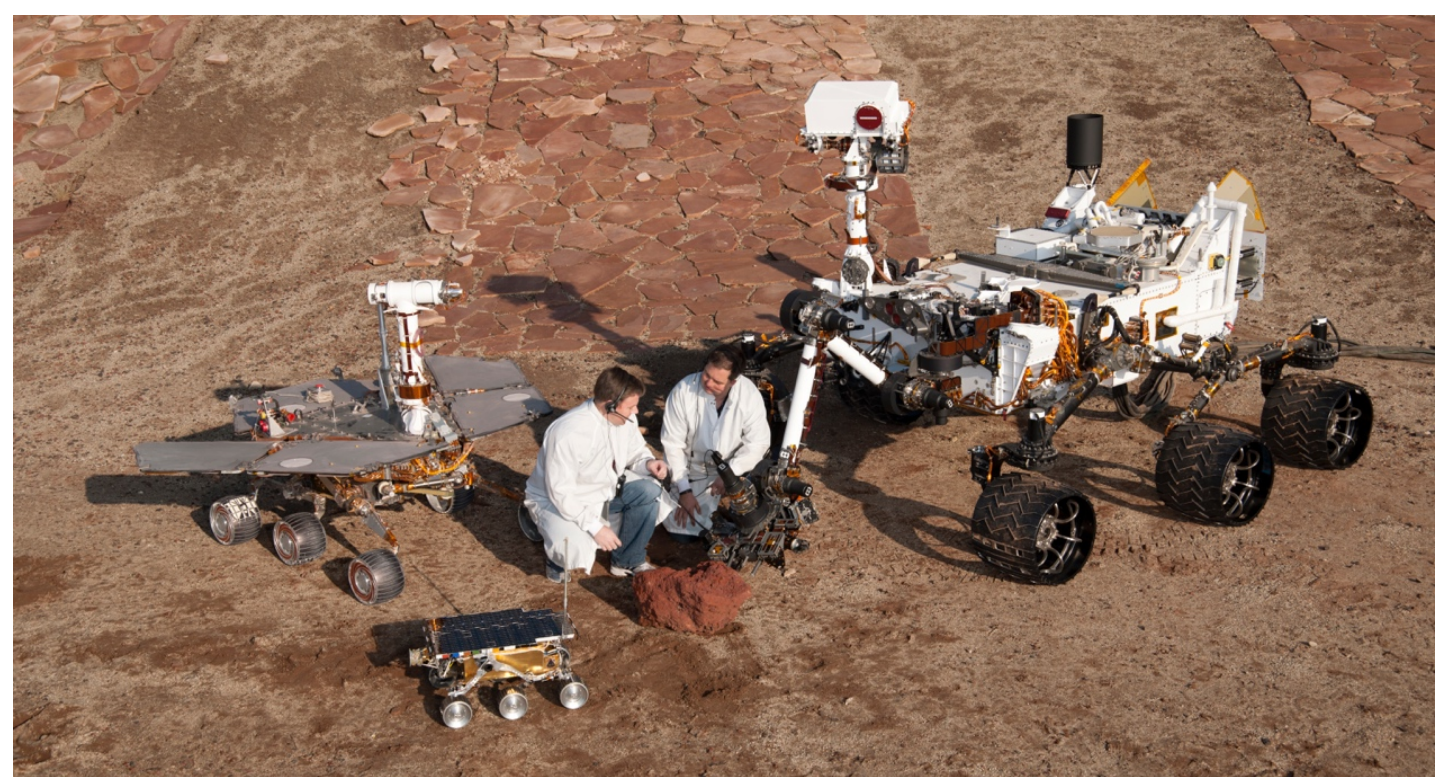

Fig. 1 One of the ways both NASA and ESA categorize their missions is by cost, basically a small, medium, or large consideration. The image shows the size of NASA's Discovery-class Mars rover (Sojourner, the smallest), the mid-sized Mars Exploration Rover (left), and the size of the Flagshipclass Curiosity rover (right). Image credit: NASA/JPL-Caltech

\subsection{ESA Mission Categories}

ESA currently (under its Cosmic Vision 2015-2025 strategic plan) has four categories of missions, which it refers to as L-Class, M-Class, S-Class, and the recently introduced F-Class. All are selected through a competitive process, though the nature of the process has evolved over time for the L-class. For example, the selection of the Jupiter Icy Moons Explorer (JUICE) mission in 2012 as the L1 mission was the result of a call for mission proposals open to all scientific themes. The scientific themes of the two subsequent L-class opportunities were identified via a "call for ideas," followed by open calls for mission proposals focused on the identified themes ("The Hot and Energetic Universe" and "The Gravitational Universe"). This has led to the Athena X-ray observatory and LISA gravitational wave observatory being selected for study and development as ESA's L2 and L3 missions. L-, M-, and S-Class missions are defined by cost. F-Class missions have both a cost and a strict time limit imposed on them. ESA's cost includes procurement of the launch vehicle and development, assembly, test, and operation of the spacecraft itself, but typically does not include the instruments or science teams. Instruments and science teams are generally funded by the individual member-states of ESA out of their national budgets, or by external countries (such as the U.S.) that decide to participate in a particular mission. Because of these external contributions, the total financial investment in an ESA mission can be much higher than its cost category. This should be kept in mind when comparing the cost-categories of NASA-led and ESA-led missions. While not a category of space mission per se, ESA's strategic plan also includes the possibility of missions of opportunity, allowing for ESA's participation on a space mission lead by another space agency within the same cost cap of S-class missions (50 M Euros).

ESA's L-Class missions are the largest and most complex, and are comparable to NASA Flagship missions, with a cost cap of $1000 \mathrm{M}$ Euros. The JUICE mission to Jupiter, described in Section 4, is an L-Class mission. ESA's M-Class missions are medium-sized, less complex than L-Class, and cost up to 550M Euros (about the same level as a NASA New Frontiers mission). 
The most recently selected M-class mission is ARIEL, to launch in mid-2028. S-Class missions are not to exceed 50M Euros in cost to ESA. Given their low cost cap, S-Class missions are not expected to be feasible for the outer solar system. In 2018 a call was made for an F-Class mission. Referred to as a fast mission opportunity, this category has a cost cap of $150 \mathrm{M}$ Euros (excluding launch vehicle and member-state contributions) and the requirement of being able to launch on (for ESA) a very short time-scale of 8 years. That time scale was selected to allow it to launch on the same launch vehicle as one of the M-class missions. The possibility of additional F-class mission calls in the future is currently under discussion. The uncertainty on the frequency of such future calls and the budget constraints of F-class missions suggests that this class is also unlikely to be relevant for giant planet exploration. In 2019 the Comet Interceptor mission was selected as the first F-class mission to fly for a shared launch with the ARIEL mission.

Compared to NASA, ESA selects missions for launch far in advance. Since 2011, ESA has selected two S-Class, $4 \mathrm{M}$-Class, and $3 \mathrm{~L}$-Class missions. While that is a large number over just 8 years, the launch dates extend out to 2034, making the pace roughly one L-Class, two $\mathrm{M}$-Class, and one S-Class per decade. Another difference between the way NASA and ESA run missions is that NASA has separate budgets and programs for planetary, astrophysical, and Earth missions. ESA, however, typically allows all science-driven missions in a cost class to compete against each other. The successor to ESA's Cosmic Vision program is currently being developed, with the community providing inputs to develop "Voyage 2050", to span from 2035 to 2050.

Table 1: Summary of NASA and ESA mission categories.

\begin{tabular}{|c|c|c|c|}
\hline Mission Class & Agency & Cost Cap $^{\text {a }}$ & Comment \\
\hline SIMPLEx/SALMON & NASA & $\$ 55 \mathrm{M}$ & Competed \\
\hline Discovery & NASA & $\$ 500 \mathrm{M}$ & Competed \\
\hline New Frontiers & NASA & $\$ 1000 \mathrm{M}$ & $\begin{array}{c}\text { Competed. Target } \\
\text { body is restricted } \\
\text { (see text) }\end{array}$ \\
\hline Flagship & NASA & N/A & Assigned \\
\hline S-Class & ESA & $50 \mathrm{M} €$ & $\begin{array}{c}\text { May not be available } \\
\text { in the future }\end{array}$ \\
\hline F-Class & ESA & $150 \mathrm{M} €$ & \\
\hline M-Class & ESA & $550 \mathrm{M} €$ & $1000 \mathrm{M} €$ \\
\hline L-Class & ESA & & \\
\hline
\end{tabular}

a Definition of mission cost varies between programs and agencies, and limits can change over time. See text.

\section{Future Missions to Gas-Giant Planets}

In this Section we discuss how approved and potential future missions may address giant planet atmospheric science objectives. Since our focus is exclusively on giant planet atmospheres, we make no attempt to describe the full science breadth of the missions.

\section{Flagship/L-Class}


There are two planned and currently funded missions to giant planets, and both are in the Flagship/L-Class category.

\section{NASA'S Europa Clipper}

NASA's Europa Clipper mission is scheduled to launch in the 2020's and arrive at Jupiter 3 to 6 years later (at the time of this writing, the launch date and launch vehicle are uncertain). It is designed for the exploration of Jupiter's moon Europa. Under current plans, there will be no scientific observations of Jupiter. None-the-less, should that policy change, Clipper could make observations to help our understanding of Jupiter's atmospheric chemistry (Mills et al. 2019) and circulation (Fletcher et al.2019).

\section{ESA's Jupiter Icy Moons Explorer (JUICE)}

Jupiter Icy Moons Explorer (JUICE) was officially selected as ESA's L1 mission in May 2012. Plans call for a launch in 2022 and arrival at Jupiter in 2029. It will be Europe's first mission to Jupiter (Grasset et al., 2013). Unlike Europa Clipper, JUICE does have scientific objectives related to Jupiter's atmosphere, in addition to ones related to three satellites (Ganymede, Callisto, and Europa) and the wider giant planet system to which these objects are connected including the magnetosphere. During its $~ 3$-year orbital tour of the Jovian system, time will be spent at higher orbital inclinations to view Jupiter's poles and high magnetic latitudes.

One of JUICE's two science themes (Grasset et al., 2013) is to study the Jupiter system as an archetype for gas giants, focusing on the atmosphere (Fig. 2). The remote sensing instruments offer the capability to sound the atmospheric structure and composition from the cloud-tops to the upper atmosphere, revealing dynamic and chemical processes that couple the different vertical domains (troposphere, stratosphere, thermosphere). The JUICE atmospheric science case was developed to be complementary with that of NASA's Juno mission, where the microwave radiometer (Janssen et al., 2017) provides access to the deep atmosphere below the clouds. The science required multi-spectral remote sensing across a broad range of wavelengths, combining high spatial resolutions at local and global scales with an orbital tour sampling a wide range of illumination conditions. Crucially, jovian atmospheric monitoring was to be a key component of the science case, requiring long-term observations to understand atmospheric variability on timescales from hours to years.

The JUICE payload suite includes instruments measuring particles and fields in the jovian environment which can influence the uppermost atmosphere (the Juice MAGnetometer, JMAG, the Particle Environment Package, PEP, and the Radio and Plasma Wave Investigation, RPWI). There are four remote sensing instruments that will directly target the atmosphere at UV, visible, near-infrared, and sub-millimeter wavelengths, and radio occultation studies will actively probe the atmosphere using the high-gain antenna. The Jovis, Amorum ac Natorum Undique Scrutator (JANUS) visible camera offers multi-band imaging in the 380-1080 nm range, using narrow-band filters selected to sound in and out of strong methane absorption bands in the jovian spectrum. The spatial resolution of JANUS will exceed the best images from the Cassini flyby ( $\sim 60 \mathrm{~km} /$ pixel), Galileo ( $\sim 15 \mathrm{~km} /$ pixel), and Juno at high latitudes ( 50 $\mathrm{km} / \mathrm{pixel})$. It will approach, but not surpass, the spatial resolution of the Juno spacecraft's JunoCam imager at the closest perijoves, simply because JUICE will not get as close to Jupiter as Juno does. JANUS will track jovian meteorological features and atmospheric winds, as well as exploring the spatial distribution of lightning on Jupiter's dark side. JANUS will be 
complemented and extended by the Moons and Jupiter Imaging Spectrometer (MAJIS) visible-near-IR spectrograph. MAJIS has two channels, one spanning 0.5-2.35 $\mu \mathrm{m}$ for reflected sunlight studies (e.g., determining the relative altitudes of cloud and haze layers), and the other spanning 2.25-5.54 $\mu \mathrm{m}$ for emission from the ionosphere $\left(\mathrm{H}_{3}{ }^{+}\right)$and midtropospheric clouds/composition. Regular scan maps of Jupiter by MAJIS will show how the clouds and composition evolve with time. At shorter wavelengths, the UV imaging Spectrograph (UVS) will offer similar capabilities to the UVS instruments on Juno and Europa Clipper, spanning the EUV and FUV from 55 to $210 \mathrm{~nm}$. This range is sensitive to absorptions from stratospheric hydrocarbons, tropospheric ammonia, and the scattering properties of aerosols, as well as airglow/aurora seen with $\mathrm{H}_{2}$ bands and Lyman-alpha emission. UVS will be used in both nadir mode for seeing emission and reflected sunlight from the atmosphere as well as in an occultation mode where it observes stars and the Sun as they pass behind the atmosphere for high-resolution vertical structure measurements. The final remote sensing instrument we discuss is the sub-millimeter instrument (SWI). It features two passively-cooled Schottky receivers working at 600 and $1200 \mathrm{GHz}$ along with ultra-high resolution spectrometers to resolve the narrow line shapes of stratospheric emission features. SWI will be able to determine the temperature and composition of Jupiter's middle atmosphere, as well as the first direct measurements of winds above the clouds via the Doppler shifting of these narrow lines.

These four remote sensing experiments will be complimented by radio occultations, using the attenuation of the JUICE signal during both ingress and egress as Jupiter moves between the Earth and the spacecraft. The attenuation at depth is due to tropospheric ammonia, but at altitudes above the clouds, this provides a sensitive way to measure vertical wave structure, temperature/density gradients, and ionospheric properties. There are some spectral gaps in the JUICE payload, such as the thermal infrared longward of $5 \mu \mathrm{m}$, which will require Earth-based resources to explore. Taken together, the JUICE instrument suite will provide a tremendous resource for furthering our understanding of the Jovian atmosphere in the 2030s.

While there are ideas and aspirations for additional Flagship-class missions to Jupiter or Saturn, as of this writing (late 2019), there do not appear to be any other leading candidates for selection in the next decade, whose science focus would include a gas-giant atmosphere. 


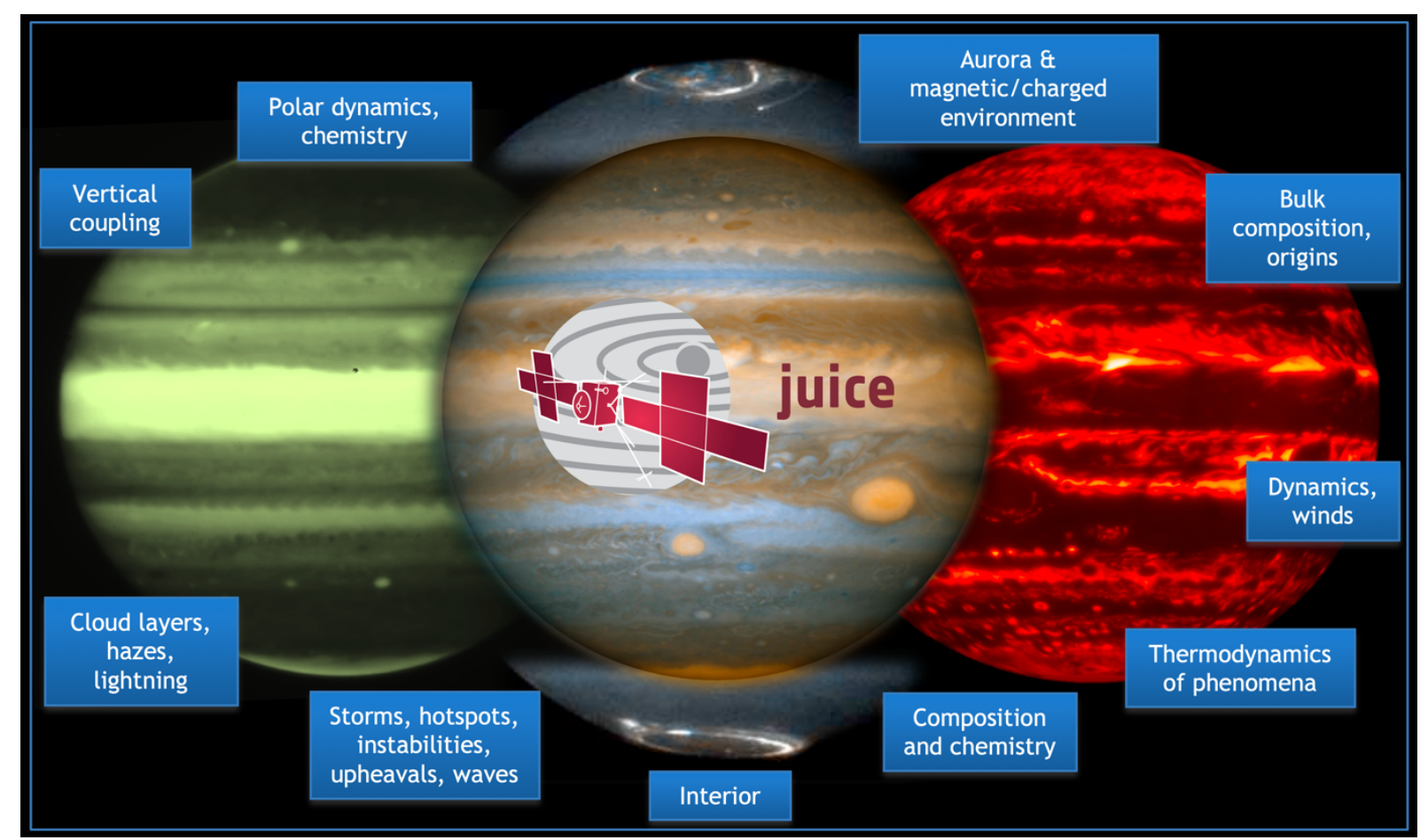

Fig. 2 Examples of the JUICE science objectives for the jovian atmosphere. Jupiter is shown in the near-infrared in reflected sunlight (left), visible (center), ultraviolet for auroral emission (top and bottom), and at 5 microns (right). The central enhanced-color image of Jupiter's clouds is from Hubble (credit: NASA/ESA/A. Simon-Miller/I. de Pater). The left- and right-hand images are from Gemini/NIRI (left in reflected sunlight, credit: Gemini Observatory/AURA/L.N. Fletcher, right in thermal emission at $5 \mu \mathrm{m}$, credit: Gemini Observatory/AURA/M. Wong). The auroral images in the UV come from Hubble (credit: NASA/ESA/J. Clarke). Montage updated from Grasset et al. (2013)

\section{New Frontiers/M-Class}

At the end of June 2019, NASA selected Dragonfly as the fourth New Frontiers mission (NF4). Led by Elizabeth Turtle of the Johns Hopkins University Applied Physics Laboratory, Dragonfly will place a rotorcraft-lander on Saturn's moon Titan. Studying Titan's thick atmosphere is one of its science objectives, but the publicly available information at the time of this writing suggests there will not be any remote sensing of Saturn's atmosphere. Since the focus of this paper is giant planet atmospheres, we will not describe this exciting mission further. Assuming the fifth New Frontiers call (expected in 2020) uses the current list of allowed missions (see Section 3.1), there are three missions potentially relevant to gas-giant planets. The Saturn atmospheric probe (Fig. 3) will clearly address highest priority gas-giant atmospheric science. The Ocean Worlds and lo Observer missions could conceivably make some contributions as well, but a major advance seems unlikely given that resources on these missions are limited and their focus is necessarily one of the satellites.

Several NASA community assessment groups, such as OPAG, have requested that NASA eliminate the restriction on targets for the New Frontiers program. It seems likely that NASA would wait until receiving the inputs of the next Planetary Science Decadal Survey before making such a change. Those inputs are not expected until 2023. Should that restriction be removed, it is expected that several New Frontiers missions would be proposed which could have impact on our understanding of gas-giant atmospheres. 
Turning to ESA's M-Class missions, a Saturn entry probe called Hera was unsuccessfully proposed for ESA's M4 and M5 competitions. The science case was outlined by Mousis et al. (2014) and the technical approach described by Mousis et al. (2016). Such a mission would make measurements to determine the bulk elemental and isotopic composition of Saturn to constrain theories of planetary formation and evolution, whilst also providing access to the thermal and compositional environment below the clouds. Given a cost cap similar to that of the New Frontiers program, one could imagine other gas-giant atmospheric missions fitting within the M-Class, but at the time of this writing the status of M-class missions beyond M5 is not clear.

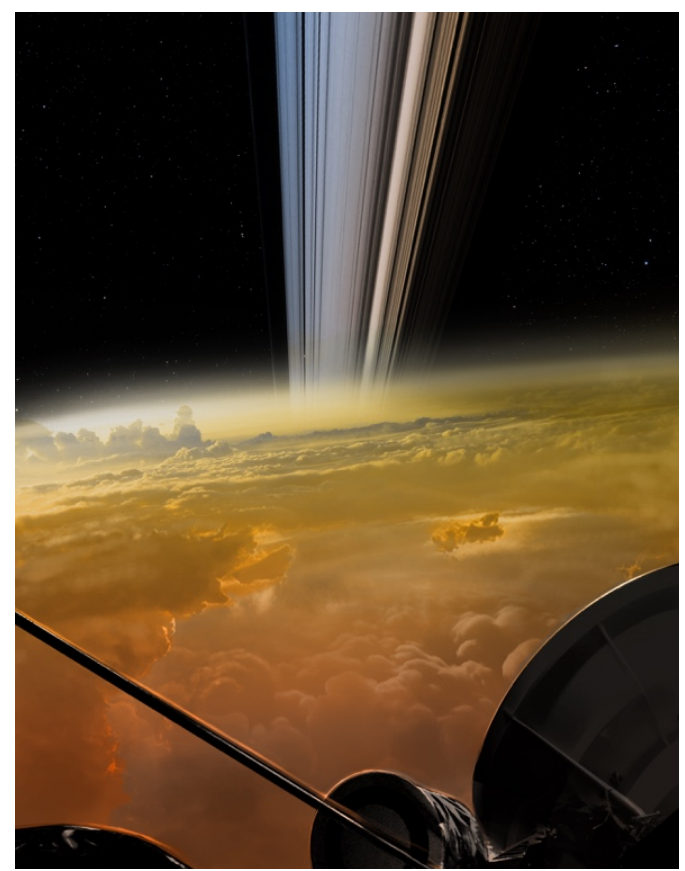

Fig. 3 Artist's conception of the view from the Cassini spacecraft as it entered Saturn's atmosphere. One of the six allowed categories of missions in NASA's New Frontiers-4 mission competition was a Saturn atmospheric probe. While that mission was not selected, a future Saturn probe will experience this view. Credit: NASA/JPLCaltech

\section{Discovery/S-Class/F-Class and Smaller Missions}

As mentioned in Section 3, there have been no successful stand-alone gas-giant missions proposed in the lowest-cost mission categories. Given the competitive nature of these programs, and the wide latitude proposers have in choosing their target and science goals, we cannot speculate on what-if any-missions may be possible in the future. We will comment that such a mission would likely have to be extremely focused in its science objectives, and limited in scope.

While it appears difficult to have entire gas-giant missions in the lowest-cost category, NASA's SALMON program has been used to successfully fund instruments and scientists to participate in ESA's JUICE mission to Jupiter (the L-Class mission described previously).

\section{Future Missions to Ice-Giant Planets}

\section{Flagship/L-Class}

There are no Flagship-Class missions to Uranus or Neptune currently approved by either NASA or ESA. The current NASA Planetary Science Decadal Survey (National Research Council 2011) calls for a Uranus orbiter and probe to be initiated as the next Flagship mission 
this decade. Budgetary realities make this unlikely, and the next Decadal Survey will identify new priorities for the 2023-2032 time frame. Since the science case for such a mission remains strong, and the outer planet community (as represented by NASA's Outer Planets Assessment Group, or OPAG) continues to support an Ice Giant mission, there is a good chance a Uranus or Neptune mission will figure prominently in the next Decadal Survey as well. Furthermore, European scientists have continued to express a strong desire for both orbital Ice Giant exploration (Arridge et al., 2012, 2014; Masters et al., 2014; Turrini et al., 2014) and in situ entry probes (Mousis et al., 2018). The Uranus Pathfinder orbital concept was proposed to both the M3 and M4 calls (Arridge et al., 2012, 2014), a Neptune mission was also proposed to M3 (OSS, Christophe et al. 2012), and Ice Giants featured strongly when ESA called for scientific themes for its L2 and L3 launch opportunities in 2013. The Ice Giant mission theme was determined to be worthy of an L-class mission, but the selection committee recommended that it be pursued in collaboration with NASA as a NASA-led mission. As a result of these ongoing efforts, discussions between NASA and ESA have occurred, and joint mission studies have already been performed (see Hofstadter et al. 2019a for a summary of a NASA-led study, while the results of an ESA-led study are described in Bayon et al. 2019). These studies highlight the existence of a number of feasible mission scenarios within the cost caps set by the two agencies. Most recently, orbital and in situ exploration of Ice Giant Systems have been proposed as a cornerstone of ESA's Voyage 20352050 program (Fletcher et al., 2019).

Should an Ice Giant mission be initiated, it is not clear if Uranus, Neptune, or both will be the target. Overall, the scientific community and mission studies have indicated that Uranus and Neptune are equally compelling objects. Uranus missions are expected to be lower cost than similar ones to Neptune (by US\$300M according to Hofstadter et al. 2019a, due to the closer distance and shorter flight times), which is attractive to both agencies. Furthermore, the optimal launch windows to Uranus occur later (2030-2032) than ones to Neptune (20282030), which allows for a longer development time and pushes peak funding needs further out, which is also attractive given existing commitments NASA and ESA have for the early 2020's (e.g. the Europa Clipper and JUICE missions). In addition to those practical reasons, there are several aspects of the Uranus system that make it a particularly intriguing science target for the study of giant planets. Foremost among these is that, unique among our solar system's giants, it is releasing almost no internal heat. This suggests a different internal structure and different energy inputs driving its atmospheric circulation (Nettelmann et al. 2013). On the other hand, the scientific communities interested in Kuiper Belt Objects and Ocean Worlds (icy satellites with sub-surface water oceans that may harbor life) favor Neptune because of the opportunity to study its moon Triton, which is a captured KBO and identified as a potential Ocean World (Hendrix et al. 2018).

Complicating the decision about which Ice Giant to target is the exciting opportunity to visit both planets utilizing two spacecraft (Turrini et al. 2014; Simon et al. 2018). (The orbital positions of the planets will not allow a single spacecraft to visit both planets-as Voyager did in the 1980's - for another 150 years.) Both the recent NASA study (Hofstadter et al. 2019a) and an Ice Giant-themed white paper for ESA's L2-L3 call (Turrini et al. 2014) emphasized that each planet has unique things to teach us, and we can only understand Ice Giants as a class of planet if we have detailed information about both Uranus and Neptune. 
The most recent ESA study (Bayon et al. 2019) has also kept open the possibility of exploring both planets, with NASA and ESA each building a spacecraft.

Regardless of which Ice Giant is visited, based on the current U.S. Planetary Science Decadal Survey (National Research Council 2011) and the more recent NASA mission study (summarized in Hofstadter et al. 2019a), the following atmospheric science goals are likely to be addressed by such a mission:

- Measure the noble gases, isotopic ratios, and abundance of species such as $\mathrm{CH}_{4}, \mathrm{NH}_{3}$, and $\mathrm{H}_{2} \mathrm{~S}$ in the upper 10 bars of the atmosphere.

- Study the three-dimensional flow in the troposphere. This includes zonal winds and meridional circulations and their vertical variations. Also look for indicators of deep convective activity in flow patterns, in the presence of disequilibrium species such as $\mathrm{CO}$ and $\mathrm{PH}_{3}$, and in the hydrogen ortho-para ratio. Finally, study temporal variations in all these parameters.

- Determine the atmospheric vertical temperature profile in the stratosphere and upper troposphere, horizontal variations in temperature, and their connections to the zonal and meridional flow.

- Measure the overall energy balance of the atmosphere (i.e. the ratio of total emitted radiation to space vs. the amount of absorbed sunlight, the difference being an indicator of the amount of internal heat entering the atmosphere from below).

- Determine upper tropospheric cloud structures (location, extent, and if possible their composition).

- Study upper atmospheric/magnetospheric interactions, including heating by charged particles and the current atmospheric loss rate.

\section{New Frontiers/M-Class}

As with the Gas Giants, there are no currently approved medium-class missions to either Uranus or Neptune. Furthermore, on the NASA side the current list of allowed New Frontiers mission does not include either planet, and during ESA's last M-Class competitions (Arridge et al. 2012, 2014), ESA determined that an Ice Giant mission was not feasible in this class by ESA alone in the framework of the current Cosmic Vision 2015-2025 strategic plan. Thus, until NASA expands its list of New Frontiers targets, it does not appear likely that a mediumclass mission will target an Ice Giant. The two agencies could potentially look to combining their independent medium-class opportunities to allow a fully collaborative mission, if a strategic/flagship-level mission to an Ice Giant is not promoted during the coming decade.

\section{Discovery/S-Class/F-Class and Smaller Missions}

As was true for the Gas Giants, it appears that among these lowest-cost missions, only NASA's Discovery program is likely to be able to support a complete Ice Giant mission, and the mission would likely need to be very limited in its scientific scope. Such a mission is, in fact, part of the current Discovery mission competition (as of the mid-2019 time of this writing), with Step-2 selection of candidates for further study due in 2020. That mission, called Trident (with Louise Prockter of the Lunar and Planetary Institute as PI), is focused on Neptune's moon Triton (Prockter et al. 2019). There is no publicly available information on whether or not any significant observations of Neptune's atmosphere will be performed. 


\section{New Technologies for Future Missions}

In Sections 4 and 5 we discussed approved as well as potential missions to the giant planets, all of which can be carried out using currently available technologies. The most recent broad survey of missions to Ice Giants for the next decade (Hofstadter et al. 2019a) also finds that the highest-priority science investigations do not require any new technologies.

Technological advances have the potential, however, to enhance the return of future missions by increasing their capabilities. Some promising examples are:

Smaller, low-power instruments

A given launch vehicle has a maximum mass it can lift, and a maximum fairing size it is designed to accommodate (the fairing is the aerodynamic shell placed around the payload to enable flight through Earth's atmosphere). Decreasing the mass and physical size of each instrument therefore allows more science instruments to be carried. Alternatively, the miniaturized instruments can be carried by a smaller spacecraft, launch vehicle, or atmospheric entry probe which decreases costs.

Power can also be a limited resource on a spacecraft, so instruments that draw less energy can allow those instruments to be operated for longer periods of time or in conjunction with additional instruments, increasing the scientific return. A low-power instrument suite also can increase the power available to the spacecraft transmitter, giving options for transmitting higher data rates or using a smaller antenna. Low-power instruments also have the potential to extend the reach of solar-powered spacecraft beyond Jupiter.

\section{Advanced radioisotope power systems and REP}

Radioisotope power systems (RPS) are the generally preferred energy source in the outer solar system, allowing for Flagship-class payloads to be operated at Uranus and beyond, for operations in eclipse, and for more maneuverable spacecraft than ones carrying large solar arrays. New RPS designs are being developed (Zakrajsek et al. 2017) with increased efficiency (reducing the amount of Plutonium needed to power a spacecraft) and increased lifetime (critically important for long-duration outer solar system missions).

Powering an ion engine with an advanced RPS (referred to as Radioisotope Electric Propulsion, or REP) can dramatically increase the capabilities of a spacecraft orbiting a giant planet. Ion engines are frequently used in the inner solar system, powered by large solar arrays (Solar-Electric Propulsion, SEP). As demonstrated by the Dawn spacecraft's ability to orbit multiple asteroids (Russel et al. 2004), REP enables flexibility in choosing orbital inclinations and radii around a giant planet, making it easier to, among other things, perform in situ observations of the uppermost atmosphere and its coupling with the rings and magnetosphere as Cassini did during its Grand Finale (Edgington and Spilker 2016; Hadid et al. 2018). It is worth noting that such orbital flexibility also enables repeated low-altitude, low velocity encounters with a giant planet's rings and satellites, or extended tours of the magnetosphere (Hofstadter et al. 2019b).

\section{Autonomous capabilities}

Given that radio signals traveling between Earth and Jupiter take more than an hour to make the round trip, having high powered on-board processors and advanced software for autonomy can enhance the science return from the outer solar system. Autonomous navigation enables extremely close flybys of targets and trajectory changes to respond to 
unexpected conditions or transient phenomena. Autonomous sequencing allows science instruments to be turned on or off and instruments pointed in response to real-time conditions. And science data can be optimally compressed or even analyzed on board drastically reducing the data volume that needs to be returned to Earth.

\section{Aerocapture}

A spacecraft encountering an outer planet arrives at relatively high velocities, making it difficult to get captured into orbit. All orbiters sent to the outer solar system so far have used large amounts of chemical fuel to slow down. The mass of the fuel, tanks, and engines limits the amount of mass available for science instruments. Furthermore, to minimize the fuel needed for orbit insertion, spacecraft are typically put on slower-than-possible trajectories which increases the time it takes to get to the outer solar system. The concept of aerocapture (Spilker et al. 2018) is to fly the spacecraft through the outer reaches of the atmosphere, where it can encounter enough drag to slow down and be captured into orbit without using any fuel. (Aerocapture should not be confused with aerobraking. Aerobraking is when atmospheric drag is used to adjust the path of an already orbiting spacecraft. Aerocapture is much more challenging, because one must lose sufficient energy on the very first pass through the atmosphere to be captured into orbit, and a mistake means your spacecraft never gets into orbit or burns up in the atmosphere.) In studies to-date (e.g. Hofstadter et al. 2019b) it is unclear whether the mass required for the aerocapture system is appreciably less than the mass associated with chemical engines and fuel, but aerocapture can reduce trip times to Neptune by several years with correspondingly smaller reductions to the nearer planets.

\section{Summary and Conclusions}

Future missions to the giant planets of our solar system will play a crucial role in increasing our understanding of the diversity of planetary atmospheres. The ability to measure the abundance and isotopic ratios of key atmospheric species (such as noble gases), which are needed to test models of the formation and evolution of these atmospheres, can only be made with an atmospheric entry probe. The higher sensitivity of measurements made from an orbiting spacecraft (relative to Earth-based instruments) allows detection of trace species important for our understanding of chemical pathways active on these planets. And the seasonal, diurnal, high-spatial, and temporal resolution offered from spacecraft, along with gravity and magnetic field measurements, allows us to better understand the dynamics and structure of these atmospheres.

Currently, NASA and ESA are the primary agencies involved in giant-planet missions. Each has one approved mission under development, both of which are large, Flagship-class projects to Jupiter. NASA's Europa Clipper mission will launch in the 2020's. Its focus is on the satellite Europa, and it is not expected to advance our understanding of gas giant atmospheres. ESA's JUICE mission (to be launched in the early 2020's) is also focused on exploration of the moons. It will, however, make extensive observations of Jupiter's atmosphere, with some instrument types that have not flown to Jupiter before (such as SWI working at sub-millimeter wavelengths). Atmospheric scientists expect to learn additional details about the upper atmosphere that will help us understand the composition and structure of gas giant atmospheres. 
There are several missions being studied which hold great promise for dramatically increasing our understanding of giant-planet atmospheres. One of the front-runners to be selected as a new NASA Flagship mission is an orbiter and atmospheric probe to be flown to one of the ice giants (Uranus or Neptune). If-as hoped by the scientific community-this is a Cassini class mission with a wide range of instruments, this will allow us for the first time to explore in depth this enigmatic class of planet, which is much different from the betterunderstood gas giants. ESA and NASA have already begun discussions and studies on flying this as a joint, NASA-led mission for launch near 2030 (Bayon et al. 2019). Options for making this a two-spacecraft effort, so that both Uranus and Neptune can be explored, are being considered (e.g. Simon et al. 2018; Bayon et al. 2019).

A mission to send an atmospheric probe into Saturn's atmosphere is also on NASA's list of possible New Frontiers missions and has been proposed to ESA's M-class program. This is an opportunity for a mid-cost mission to make significant contributions to our understanding of the diversity of atmospheres by allowing us to compare noble gas abundances, isotopic ratios, and the abundance of other species in Saturn's atmosphere with the composition of Jupiter's atmosphere as measured by the Galileo probe in 1995. While not selected in either the most recent New Frontiers competition (NF-4) or the European M5 competition, it is expected that there will be another opportunity to propose this mission in the coming decade. Saturn's moons Enceladus and Titan also appear in the current list of possible New Frontier targets, but those missions are unlikely to make significant contributions to Saturn atmospheric science. If NASA elects to expand the list of allowed New Frontiers targets (something recommended by several of its community-based Assessment Groups), it is likely that additional giant-planet missions will be proposed, some of which include significant atmospheric-science investigations.

NASA's Discovery program also provides an opportunity to send spacecraft into (or at least fly through) a giant-planet system. These low-cost missions need to have very focused science objectives. In the competition underway at the time of this writing (Discovery 15/16, with selections expected in 2021) there are no missions focused on giant-planet atmospheres. It is certainly possible, though, for future Discovery proposals to make important contributions to atmospheric science. NASA's lowest-cost programs (SIMPLEx/SALMON) are not able to support giant-planet missions, though they can provide for U.S. instruments or scientists to participate in a mission flown by a non-U.S. space agency.

On the ESA side of things, looking ahead as far as 2035 (the limit of their current long-term plan, referred to as Cosmic Vision) there are no further ESA-led missions to the giant planets beyond JUICE, although ESA has clearly expressed interest in partnering on NASA-led efforts. ESA is in the early stages of developing its "Voyage 2050" plan for the period from 2035 to 2050 , and both in situ entry probes for the giant planets and orbital exploration of the Ice Giants are being proposed for that long-term program.

This paper has focused on the planetary spacecraft that will advance our understanding of giant planets, but these missions do not operate in isolation. Remote sensing from both ground-based facilities and space-based observatories can provide complementary and synergistic science, providing longer temporal baselines, monitoring for transient 
phenomena (e.g. impacts, Hueso et al. 2019), different spectral ranges, and global coverage to support regional imaging. The coming decade will see the launch of the James Webb Space Telescope, providing spatial and spectral coverage across the infrared from 1 to $30 \mu \mathrm{m}$, capturing reflected sunlight, ionospheric/auroral emission, and thermal emission from the atmospheres of all four giants (Norwood et al., 2016). A guaranteed-time program is in place that will target Jupiter's Great Red Spot and polar domains, Saturn's summertime hemisphere and polar vortex, and provide global thermal maps of Uranus and Neptune. An early-release science program will also provide further observations of the Jovian system as a test-case for the capabilities of JWST, particularly for the observation of large, bright, rotating, and ever-changing objects. Future space observatories such as the Origins Space Telescope (in the sub-millimeter range) and LUVOIR (in the optical-UV) could provide new measurements of the giant planets, but the instruments will not be optimized for them. Novel new concepts for dedicated time-domain observations of the giant planets (e.g., Wong et al., 2009, Bell et al., 2015) have the potential to revolutionize our understanding of these worlds, but none have yet progressed from the proposal stage. Finally, the 2020s will see the first-light for the extremely large optical telescopes, with primary mirrors some $30-40 \mathrm{~m}$ in diameter, capable of providing exquisite spatial resolution of the giant planet systems. Each of these could deliver new insights into the diversity of planetary atmospheres to complement the spacecraft missions described here.

While budgetary and programmatic considerations necessarily limit how much each space agency can do at the giant planets, the above discussions indicate that there are several scientifically compelling mission ideas for giant planet atmospheres which can fly. Community support, such as participating on proposal teams, writing White Papers for NASA's Decadal Survey or ESA's Voyage 2050 efforts, or speaking at public forums (such as OPAG), will help ensure the critical atmospheric science that requires space missions is achieved. 


\section{References:}

Arridge, C. S., Achilleos, N., Agarwal, J., et al. (2014), The science case for an orbital mission to Uranus: Exploring the origins and evolution of ice giant planets, Planetary and Space Science, 104, 122, (doi:10.1016/j.pss.2014.08.009).

Arridge, C. S., Agnor, C. B., André, N., et al. (2012), Uranus Pathfinder: exploring the origins and evolution of Ice Giant planets, Experimental Astronomy, 33, 753, (doi:10.1007/s10686011-9251-4).

Atreya, S.K., Crida, A., Guillot, T., Lunine, J.I., Madhusudhan, N., Mousis, O., 2019. The origin and evolution of Saturn, with exoplanet perspective. In: Baines, K., Flasar, M., Krupp, N., Stallard, T. (Eds.), Saturn in the 21st Century. Cambridge University Press, pp. 5-43.

Bayon S., et al. 2019, Ice Giants CDF Study Report, ESA document CDF-187(C), report available at the following link: http://sci.esa.int/jump.cfm?oid=61307.

Bell, J. F., Schneider, N. M., Brown, M. E., et al. (2015), Kuiper: A Discover - Class Observatory for Outer Solar System Giant Planets, Satellites, and Small Bodies, Spacecraft Reconnaissance of Asteroid and Comet Interiors, 1829, 6043, (doi:).

Christophe, B., Spilker, L.J., Anderson, J.D. et al. (2012). OSS (Outer Solar System): a fundamental and planetary physics mission to Neptune, Triton and the Kuiper Belt, Experimental Astronomy 34, 203, (doi:10.1007/s10686-012-9309-y).

Edgington, S.G., Spilker, L.J. (2016), Cassini's Grand Finale, Nature Geoscience 9, 472-473.

Fletcher et al. (2019), Ice Giant Systems: The Scientific Potential of Missions to the Uranus and Neptune Systems, White Paper submitted to ESA's Voyage 2050 Call.

Fletcher, L., Kaspi, Y., Showman, A., Guillot, T. (2019), How Well do we Understand the Belt/Zone Circulation of giant Planet Atmospheres?, Sp. Sci. Reviews, this issue.

Gaillard, F., Noack, L., Bouhifd, M., Furi, E., et al. (2019), Secular Evolutions in Planetary Degassing: Source and Geodynamic Controls on Volatile-Transfers, Sp. Sci. Reviews, this issue.

Grasset, O., Dougherty, M. K., Coustenis, A., et al. (2013), JUpiter ICy moons Explorer (JUICE): An ESA mission to orbit Ganymede and to characterise the Jupiter system, Planetary and Space Science, 78, 1, (doi:10.1016/j.pss.2012.12.002).

Hadid, L.Z., Morooka, M.W., Wahlund, J.-E., Persoon, A.M., Andrews, D.J., Shebanits, O., Kurth, W.S., Vigren, E., Edberg, N.J.T., Nagy, A.F., Eriksson, A.I. (2018), Saturn's lonopshere: Electron Density Altitude Profiles and D Ring Interaction from the Cassini Grand Finale, $G R L$ 46, 9362-9369 (doi: 10.1029/2018GL078004). 
Hueso, R., Delcroix, M., Sánchez-Lavega, A., Pedranghelu, S., Kernbauer, G., McKeon, J., Fleckstein, A., Wesley, A., Gómez-Forrellad, M., Rojas, J.F., Juaristi, J. (2018), Small Impacts on the Giant Planet Jupiter, Astronomy and Astrophysics, 617, A68.

https://doi.org/10.1051/0004-6361/201832689.

Hofstadter, M., Simon, A., Atreya, S., Banfield, D., Fortney, J.J., Hayes, A., Hedman, M., Hospodarsky, G., Mandt, K., Masters, A., Showalter, M., Soderlund, K.M., Turrini, D., Turtle, E., Reh, K., Elliott, J., Arora, N., Petropoulos, A., and the Ice Giant Mission Study Team, 2019a. Uranus and Neptune missions: A study in advance of the next Planetary Science Decadal Survey. Plan. Sp. Sci., 177. https://doi.org/10.1016/j.pss.2019.06.004.

Hofstadter, M.D., Austin, A., Chmielewski, A.B., Cutts, J.A., Elia, P., Elliott, J.O., Frazier, W.E., Hofgartner, J.D., Polit-Casillas, R., Strange, N.J., Reh, K.R., Wilcox, B.H. (2019b), Ice Giant Missions Enabled by New Technologies--or--Not Your Parent's Ice Giant Mission, Abstract P34C-08 presented at 2019 AGU Fall Meeting, San Francisco, CA, 9-13 Dec. Available on-line at https://agu.confex.com/agu/fm19/meetingapp.cgi/Paper/607916.

Hendrix, A.R. and 27 co-authors, 2018. The NASA roadmap to ocean worlds. Astrobiology 19, 1-27. https://doi.org/10.1089/ast.2018.1955.

Janssen, M. A., Oswald, J. E., Brown, S. T., et al. (2017), MWR: Microwave Radiometer for the Juno Mission to Jupiter, Space Science Reviews, 213, 139, (doi:10.1007/s11214-017-0349-5).

Leconte, J., Bolmont, E., Charnay, B., Forget, F., Gaillard, F., Godolt, M., Grenfell, L., Noack, L., Rauer, H., Tian F., Turbet, M. (2019), Could there be Exo-Atmospheres Drastically Different from what is Found in the Solar System?, Sp. Sci. Reviews, this issue.

Masters, A., Achilleos, N., Agnor, C. B., et al. (2014), Neptune and Triton: Essential pieces of the Solar System puzzle, Planetary and Space Science, 104, 108, (doi:10.1016/j.pss.2014.05.008).

Mills, F., Moses, J., Gao, P., Tsai, S-M., et al. (2019), The Diversity of Planetary Atmospheric Chemistry: Lessons and Challenges from our Solar System and Extrasolar Planets, Sp. Sci. Reviews, this issue.

Mousis, O., Atkinson, D. H., Cavalié, T., et al. (2018), Scientific rationale for Uranus and Neptune in situ explorations, Planetary and Space Science, 155, 12, (doi:10.1016/j.pss.2017.10.005).

Mousis, O., Atkinson, D. H., Spilker, T., et al. (2016), The Hera Saturn entry probe mission, Planetary and Space Science, 130, 80, (doi:10.1016/j.pss.2015.06.020).

Mousis, O., Fletcher, L. N., Lebreton, J.-P., et al. (2014), Scientific rationale for Saturn's in situ exploration, Planetary and Space Science, 104, 29, (doi:10.1016/j.pss.2014.09.014). 
National Research Council, 2011. Vision and Voyages for Planetary Science in the Decade 2013-2022. The National Academies Press, Washington, D.C.

https://doi.org/10.17226/13117.

Nettelmann, N., Helled, R., Fortney, J.J., Redmer, R., 2013. New indication for a dichotomy in the interior structure of Uranus and Neptune from the application of modified shape and rotation data. Plan. Sp. Sci. 77, 143-151.

Norwood, J., Moses, J., Fletcher, L. N., et al. (2016), Giant Planet Observations with the James Webb Space Telescope, Publications of the Astronomical Society of the Pacific, 128, 018005, (doi:10.1088/1538-3873/128/959/018005).

Prockter, L. M., Mitchell, K. L., Howett, C. J. A., et al. (2019), Exploring Triton with Trident: A Discovery Class Mission, Lunar and Planetary Science Conference, 3188, (doi:).

Russel, C.T., Coradini, A., Christensen, U., De Sanctis, M.C., Feldman, W.C., Jaumann, R., Keller, H.U., Konopliv, A.S., McCord, T.B., McFadden, L.A., McSween, H.Y., Mottola, S., Neukum, G., Pieters, C.M., Prettyman, T.H., Raymond, C.A., Smith, D.E., Sykes, M.V., Williams, B.G., Wise, J., Zuber, M.T. (2004), Dawn: A Journey in Space and Time, Plan. Sp. Sci 52, 465-489 (doi: 10.1016/j.pss.2003.06.013).

Showman, A.P. (2019), Atmospheric Dynamics of Warm and Hot Giant Planets, Sp. Sci. Reviews, this issue.

Simon, A. A., Stern, S. A., \& Hofstadter, M. (2018), Outer Solar System Exploration: A Compelling and Unified Dual Mission Decadal Strategy for Exploring Uranus, Neptune, Triton, Dwarf Planets, and Small KBOs and Centaurs, arXiv e-prints, arXiv:1807.08769, (doi:).

Spilker, T.R., Adler, M., Arora, N., Beauchamp, P.M., Cutts, J.A., Munk, M.M., Powell, R.W., Braun, R.D., Wercinski, P.F. (2018), Qualitative Assessment of Aerocapture and Applications to Future Missions, Jour. Spacecraft and Rockets 56 (doi: 10.2514/1.A34056).

Turrini, D., Politi, R., Peron, R., et al. (2014), The comparative exploration of the ice giant planets with twin spacecraft: Unveiling the history of our Solar System, Planetary and Space Science, 104, 93, (doi:10.1016/j.pss.2014.09.005).

Wong, M. H., Ádámkovics, M., Benecchi, S., et al. (2009), A Dedicated Space Observatory For Time-domain Solar System Science, AAS/Division for Planetary Sciences Meeting Abstracts \#41, 16.09.

Zakrajsek, J.F., Hamley, J.A., Sutliff, T.J., McCallum, P.W., Sandifer, C.E., Bishop, T., McCune, M.M., Cairns-Gallimore, D.A. (2017), Radioisotope Power Systems Program Status and Expectations, 15th Int. Energy Conversion Engineering Conference (doi: 10.2514/6.20174609). 